\title{
Investigation of the Validity of an Experimental Model for the Estimated Shear Force on Buttocks in a Comfortable Sitting Posture
}

J. Phys. Ther. Sci.

20: 157-162, 2008

\author{
Kenichi Kobara, PT, MS ${ }^{1,2)}$, Atsuko Eguchi, PT, $\mathrm{PhD}^{1)}$, Susumu Watanabe, PT, $\mathrm{PhD}^{1)}$, \\ YUICHI ISHIURA, OT, MS ${ }^{1}$, DAISUKE FUJITA, PT, MS ${ }^{1)}$, TETSUYA Nishimoto, PT $^{1)}$, \\ KOICHI SHINKODA, PT, $\mathrm{PhD}^{3)}$ \\ ${ }^{1)}$ Department of Rehabilitation, Faculty of Health Science and Technology, Kawasaki \\ University of Medical Welfare: 288 Matsushima, Kurashiki, Okayama 701-0193, Japan. \\ TEL +81 86-462-1111 \\ ${ }^{2)}$ Doctoral Program, Graduate School of Health Sciences, Hiroshima University \\ ${ }^{3)}$ Graduate School of Health Sciences, Hiroshima University
}

\begin{abstract}
Purpose] The purpose of the present study was to prepare an experimental model for estimating the shear force for a person sitting in a comfortable posture while leaning against a backrest, and then to verify its validity. [Subjects] The subjects were 16 healthy adult men without leg and trunk diseases (age: $21.6 \pm 0.8$ years; height: $172.5 \pm 5.3[\mathrm{~cm}]$; and body weight: $63.4 \pm 6.4[\mathrm{~kg}]$ ). [Methods] Using the model, the shear force applied to the gluteal region in a sitting posture was estimated, and at the same time, the shear force was measured using a force plate. The model's validity was assessed by comparing the estimated and measured values in terms of correlation coefficients. [Results] The average estimated shear force was $36.6 \pm 11.8[\mathrm{~N}]$, and the average reaction force in the posterior direction as part of the anteroposterior components of the floor reaction force (shear force in the anterior direction) was $35.9 \pm 15.8$ [N]. No significant difference existed ( $p=0.797)$, and the correlation coefficient was $0.897(\mathrm{p}<0.0001)$, thus exhibiting a strong positive correlation. [Conclusion] This result shows that the validity of the present experimental model is high, and the model is useful in clinical settings because shear force in a comfortable sitting posture can be relatively easily estimated without a special device.
\end{abstract}

Key words: Experimental model, Comfortable sitting, Estimated shear force

(This article was submitted Jan. 28, 2008, and was accepted Mar. 17, 2008)

\section{INTRODUCTION}

The results of recent studies on the pressure ulcers have clarified that complex stresses are generated inside the body. Not only pressure force, but also shear force, acts on the skin surface (surface shear force) ${ }^{1)}$. While the risk for pressure ulcer onset was once considered to be determined by "pressure $\times$ time", it is now thought to be regulated by "stress (pressure stress, shear stress and tensile stress) $\times$ time $\times$ frequency" $"$.
In recent years, in order to prevent people from becoming bedridden, patients have been forced to sit in wheelchairs for long periods of time ${ }^{2)}$. Also, for some old people with leg disorders, such as poststroke hemiplegia patients and patients with spinal cord injury, the amount of time spent in a chair is much longer than that of healthy individuals. However, those patients do not sit in different positions, and they tend to alleviate uncomfortable compression around the ischial bone by leaning against the backrest to increase the area of support. 
Here, the friction force between the seating surface and the skin in the gluteal region does not cause a marked positional shift in the pelvic area, but shear force is generated causing muscle and soft tissue deformations near the ischial and sacral bones ${ }^{3)}$. Gilsdorf et al. ${ }^{4)}$ and Goossens et al. ${ }^{5)}$ reported that, because shear force was generated when reclining in a wheelchair, relieving concurrent shear force was necessary for the prevention of pressure ulcers. For these reasons, it is clinically significant to investigate shear force from the standpoint of preventing pressure ulcers.

Therefore, in the present study we considered the spinal column posterior inclination and head repositioning, and an experimental model for the body section above the pelvis was prepared to estimate the shear force while sitting comfortably in a chair and leaning against a backrest. The values estimated using the model and the values measured using a force plate were compared to verify the model's validity.

\section{SUBJECTS AND METHODS}

The subjects were 16 healthy adult men without leg and trunk diseases (age: $21.6 \pm 0.8$ years; height: $172.5 \pm 5.3[\mathrm{~cm}]$; and body weight: $63.4 \pm 6.4[\mathrm{~kg}])$. In order to estimate shear force in a comfortable sitting posture, seating height $(93.4 \pm 2.4[\mathrm{~cm}])$, total head height (from head vertex to gnathion: $22.8 \pm 1.0[\mathrm{~cm}]$ ) and neck length (from lower edge of occipital protuberance to seventh cervical spinous process: $11.3 \pm 0.8[\mathrm{~cm}])$ were measured. The study objective, significance, methods, and privacy protection were explained to the subjects in writing, and each participant provided informed written consent.

In the present study, in order to eliminate the effects of the legs, a comfortable sitting posture was defined as the long sitting posture while leaning against a backrest. Also, in order to eliminate the cushioning effects of the backrest and the friction with the back, a metal parallel bar (OG GIKEN, Japan) was used as a backrest. Furthermore, in order to accurately measure the amount of force applied to the gluteal region, the parallel bar was positioned horizontally without touching the force plate.

When measuring the amount of force applied to the gluteal region while sitting comfortably, a force plate (Anima Corp., Japan, FORCE PLATE MG-
1120) was used to measure the reaction force in the posterior direction as the shear force in the anterior direction. The sampling frequency was $60[\mathrm{~Hz}]$, and during 2 -second measurements, the average value was calculated for the middle one second $(0.5-1.5 \mathrm{sec})$. The measurement posture was the long sitting position in which the gluteal region was placed on the force plate with the legs positioned outside the plate. The parallel bar was positioned at the back of the body as a backrest, at a height of 43 $\mathrm{cm}$ from the floor. In the basic sitting posture, the gluteal region was positioned in the anteroposterior mid-position of the body trunk and pelvis so that the back lightly touched the backrest. The comfortable sitting posture was defined as that in which the subject leaned against the parallel bar, $5 \mathrm{~cm}$ forward from the position of basic sitting posture not to become the sacral sitting posture ${ }^{3)}$. In order to make friction with the floor and clothing constant, all subjects wore the same clothing material. Furthermore, in order to make it easy to consider the effects of leg friction, the left and right lower legs were placed on a roller board (Sakai Medical Co., Ltd, Japan, DYJOC Board, caster diameter $40 \mathrm{~mm}$ ). Each subject was instructed to place the arms on the thighs in a relaxed state (Fig. 1).

The experimental model described below did not consider the effects of the legs. Therefore, in order to investigate the correlation between the measured and estimated values, it was necessary to eliminate not only the friction between the legs and the floor, but also the friction between the casters and the floor, from the shear force estimated using the experimental model. In order to calculate the static friction coefficient between the casters and floor, the maximum static friction force was measured. For measurement of the maximum static friction force, a digital balance (Custom Co., Ltd, Japan, HANDY SCALE) and 20 and 40 [kg] weights were used. The roller board with these weights was pulled using a leather belt in the horizontal direction in relation to the floor, and the force was measured using the digital scale immediately after the board started to move. With each weight, a total of three measurements were taken, and the average value was calculated as the maximum static friction force. By dividing the maximum static friction force by the vertical force with the weight of the board and the added weights (235 and $431[\mathrm{~N}])$, the static friction coefficient $(\mu=0.1)$ between the casters and floor was calculated. 


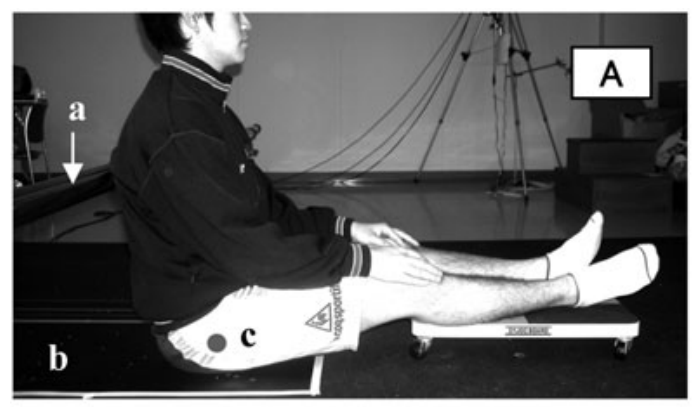

A: Basic sitting posture

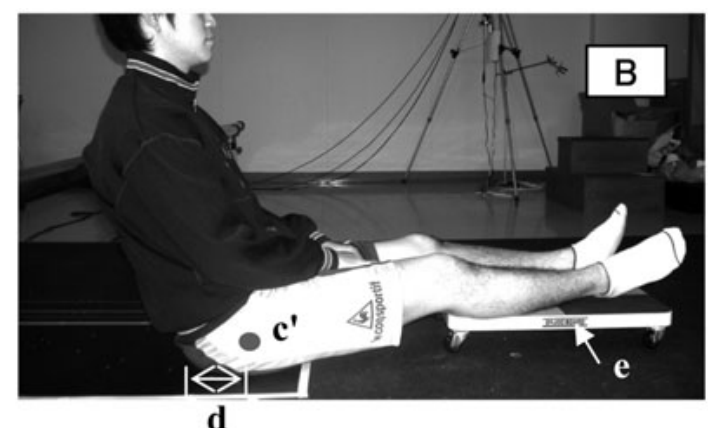

B: Comfortable sitting posture

Fig. 1. Sitting posture.

a) Parallel bar (43 cm from the floor). Placed in the horizontal direction without placing on the force plate. b) Force plate. c) Position of the greater trochanter in the basic sitting posture. c') Position of the greater trochanter in the comfortable sitting posture. d) Difference between c and c' positions: $5 \mathrm{~cm}$. e) Roller board (caster diameter: $40 \mathrm{~mm}$ )

In the present study, as shown in Fig. 2, the upper body was divided in the sagittal plane into head, neck and trunk (including the pelvis) segments, and the weight and center of mass (COM) of each segment were calculated based on body measurements and anatomical data ${ }^{6}$. The backward curvature of the spinal column caused by leaning back against the backrest resulted in mild flexure of the body trunk. Therefore, the COM of the trunk was set along the body side by taking into account the mild flexion. Also, the COM of the head and neck segments were set after repositioning from the posterior inclination, and the COMs of the three segments were determined according to the method of Kubo et al. ${ }^{7)}$. The following were measured: the angle formed by the floor and the line connecting the resultant COM and the ischial bone $(\alpha)$; the distance between the resultant COM and the intersection point between the contact point on the backrest and the line perpendicular to the line connecting the resultant COM and the ischial bone $\left(l_{1}\right)$; and the distance between the resultant gravity position and ischial bone $\left(l_{2}\right)$. Furthermore, the angle between the floor and the line between the ischial bone and the contact point between the back and backrest $(\beta)$ was measured. These anthropometric data were measured using a goniometer and an anthropometer. Estimated values were calculated by entering these values into the experimental model described below.

Next, the experimental model for estimating the shear force in a comfortable sitting posture is explained. First of all, the acting force of the upper body caused by the posterior inclination of the trunk

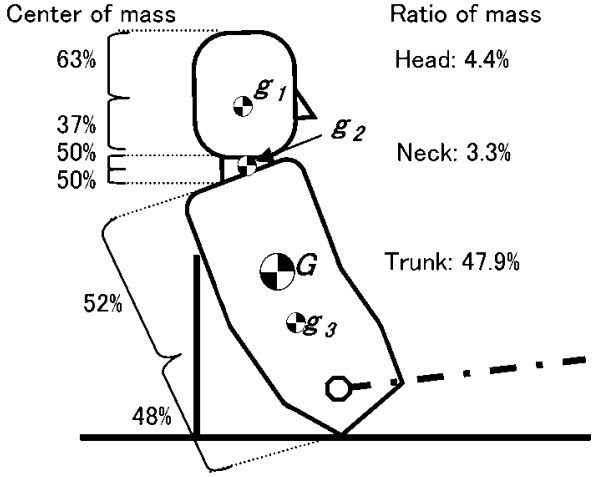

Fig. 2. COM and resultant position of each body segment. $g_{1}$ : COM of head, $g_{2}$ : COM of neck, $g_{3}$ : COM of trunk, $G$ : resultant COM of each segment. The upper body was divided into three segments in the sagittal plane: head, neck and trunk (including the pelvis) segments. The weight and mass center of each segment were calculated based on body measurements and anatomical data (Nakamura, et al. 2005). Formula for the weight and COM of each body segment.

$W_{n}$ : Weight of each body segment $(\mathrm{kg})$.

$W$ : body weight $(\mathrm{kg})$.

$W_{n}=\mathrm{W} \times$ mass ratio in relation to body weight (\%). $g_{n}$ : COM from the upper edge of each body segment $(\mathrm{cm})$.

$L$ : Length of each body segment $(\mathrm{cm})$.

$g_{n}=\mathrm{L} \times$ COM position ratio from the upper edge (\%).

and pelvis was calculated. As shown in Fig. 3A, by defining the angle between the floor and the line connecting the ischial bone and the resultant COM as $\alpha$, the vector orthogonal to the line between the ischial bone and the resultant COM, $X$, for the upper body weight, $W$, can be expressed as follows: (Formula 1).

$$
X=W \times \cos \alpha
$$




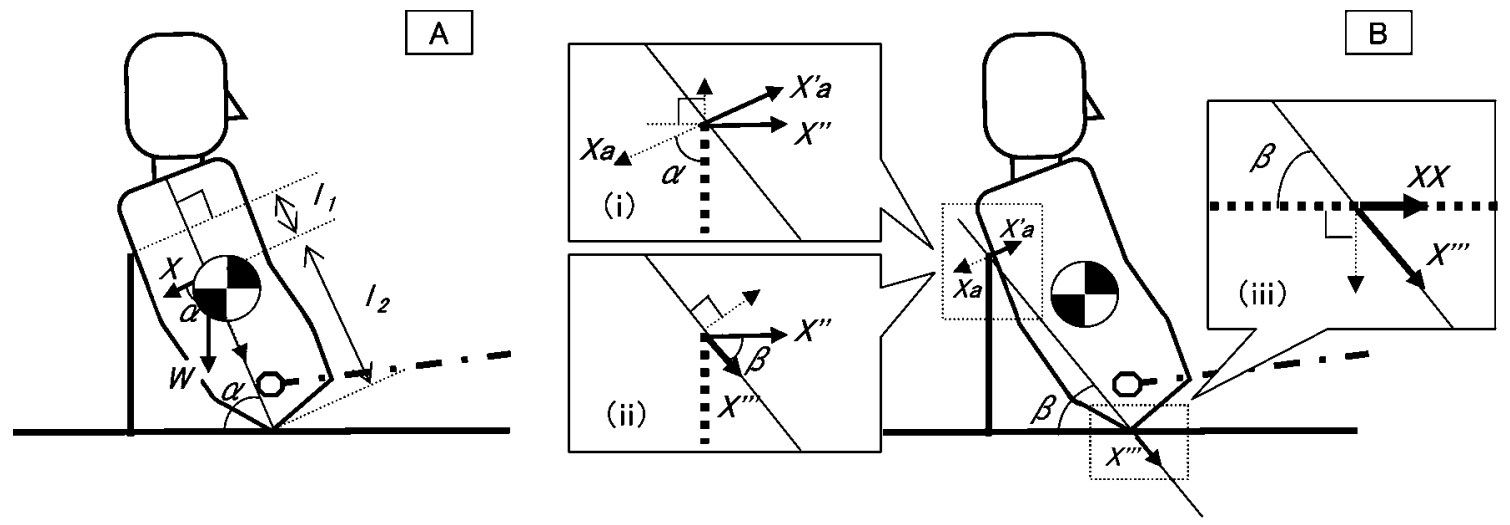

Fig. 3. A rigid link model of the upper body.

A. Acting force of the upper body accompanying the posterior inclination of the trunk and pelvis

$l_{1}$ : The distance between the resultant COM and the intersection point between the contact point on the backrest and the line perpendicular to the line connecting the resultant COM and the ischial bone $(11.0 \pm 4.2 \mathrm{~cm})$.

$l_{2}$ : The distance between the resultant COM position and ischial bone $(37.9 \pm 4.6 \mathrm{~cm})$.

$\alpha$ : The angle formed by the floor and the line connecting the resultant COM and the ischial bone $\left(56.7 \pm 3.8^{\circ}\right)$

$W$ : Body weight of the upper body above the pelvis.

$X$ : Vector orthogonal to the line connecting the resultant COM and the ischial bone.

B. Shear force estimated based on the reaction force from the contact point with the backrest.

$\beta$ : The angle between the floor and the line between the ischial bone and the contact point between the back and backrest $\left(48.8 \pm 2.3^{\circ}\right)$.

$X a$ : Acting force of the upper body applied to the backrest.

$X a$ ': Reaction force from the contact point with the backrest.

$X$ ': Anterior component of $X a$ '.

$X$,': Parallel component of the line connecting the ischial bone and the contact point with the backrest for X".

$X X$ : Estimated shear force.

Next, " $l_{l}$ " is defined as the distance between the resultant COM and the intersection point between the vertical line from the point of contact at the backrest and the line connecting the ischial bone and the resultant COM, and " $l_{2}$ " is defined as the distance between the ischial bone and the resultant COM. According to the leverage principle, leaning against the backrest generates a force, $X a$, at the contact point between the back and backrest, as expressed by Formula (2). According to the actionreaction law, the reaction force, $X a^{\prime}$, which is the same as $X a$, is generated from the contact point with the backrest (Fig. 3B-i).

$$
X a=l_{2} /\left(l_{1}+l_{2}\right) \cdot X=X a \text { ' }
$$

As shown in Fig. 3B-i, when dividing the reaction force from the contact point with the backrest, $\mathrm{Xa}$, into vertical and anterior components, the force in the anterior direction, $X$ ", can be expressed as follows: (Formula 3).

$$
X^{\prime \prime}=X a^{\prime} \sin \alpha
$$

Furthermore, as shown in Fig. 3B-ii, by defining the angle between the floor and the line connecting the ischial bone and the contact point between the back and the backrest as $\beta$, when dividing the force in the anterior direction, $X$ ", into the parallel direction of the line connecting the ischial bone and the contact point with the backrest and the line perpendicular to the parallel line, the force in the parallel direction, $X^{\text {," , }}$, can be expressed as follows: (Formula 4).

$$
X^{\prime, '}=X^{\prime \prime} \cos \beta
$$

The force from the backrest, $X$,', becomes the force in the lower anterior direction applied to the ischial bone via the body trunk. As shown in Fig. 3B-iii, when dividing this force into the vertical and anteroposterior directions, the force in the anterior direction, $X X$, can be expressed as follows (Formula $5)$.

$$
X X=X^{\prime, '} \cos \beta
$$

The value calculated by this formula, $X X$, is the estimated shear force value.

The values estimated by the present experimental 
model do not take into account the effects of leg friction. Also, the shear force applied to the gluteal region and the leg friction forces are vectors in opposite directions. The maximum static friction force is calculated by multiplying the above mentioned static friction coefficient between the floor and casters and the leg weight. Therefore, before investigating the validity of the estimated shear force values, the maximum static friction force $(19.9 \pm 2.0[\mathrm{~N}])$ was subtracted. The validity of the experimental model was verified by comparing actual force plate measurements and the values determined by subtracting the maximum static friction force from the estimated values.

In order to investigate the validity of the experimental model, we compared the estimated shear force with the measured shear force. For statistical analysis, a paired t test and Pearson rank correlation were used with the level of significance determined as $\mathrm{p}<0.0001$. The statistical analyses were performed using the Statistical Package for the Social Sciences (SPSS) ver. 14.0J for Windows.

\section{RESULTS}

The reaction in the posterior direction as part of the anteroposterior component of the floor reaction force (shear force in the anterior direction) was 35.9 $\pm 15.8[\mathrm{~N}]$, and the value after the maximum static friction force was subtracted from the estimated shear force was $36.6 \pm 11.8[\mathrm{~N}]$. There was no significant difference between the two values $(\mathrm{p}=0.797)$; thus, there was a strong positive correlation $(r=0.897, p<0.0001)$ (Table 1, Fig. 4).

\section{DISCUSSION}

While a person is sitting comfortably, some shear force is generated between the seating surface and the gluteal region. With compressive pressure, the shear force acts on the skin surface causing various stresses in the body which act to form pressure ulcers ${ }^{1)}$. In order to investigate the relationship between compressive pressure and shear force, Sakuta et al. ${ }^{8)}$ measured the changes in blood flow due to these loads. Their results suggest that 50 $\mathrm{mmHg}$ of pressure and $0.9 \mathrm{~N} / \mathrm{cm}^{2}$ of shear force are generally equivalent in biological soft tissue, and their study also showed the importance of reducing shear force in order to prevent pressure ulcers from the viewpoint of blood flow. Nojima et al. ${ }^{9)}$ also
Table 1. Comparion of the estimated and measured values

$\mathrm{n}=14$

\begin{tabular}{cc}
\hline $\begin{array}{c}\text { Measured shear force } \\
35.9 \pm 15.8\end{array}$ & $\begin{array}{c}\text { Estimated shear force } \\
36.6 \pm 11.8\end{array}$ \\
& $\mathrm{p}=0.797$ (paired t-test).
\end{tabular}

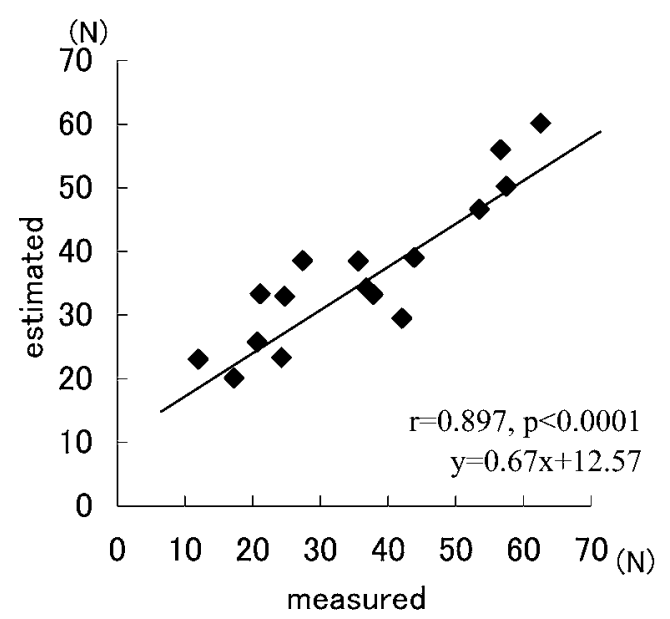

Fig. 4. Correlation of the estimated and measured values.

investigated in vivo stress by adding pressure and shear force using a biological gluteal model and a cushion model, and they reported that compared to pressure alone, shear force and pressure increased local shearing distortion in the body. In recent years, from the viewpoint of pressure ulcer prevention, many studies have been conducted on shear force. The risk of a pressure ulcer in the sacral and caudal regions due to long-term sitting is high for some institutionalized residents, and one study documented the effectiveness of seating guidance for the prevention of pressure ulcers ${ }^{10)}$. Seating guidance for prevention of pressure ulcers is beginning to be recognized as one of the roles of physical therapists, and for effective seating guidance, analysis of sitting postures is important. However, the number of studies on sitting postures and shear force remains $\operatorname{low}^{4,5)}$. Therefore, in the present study, an experimental model was prepared to estimate shear force while sitting comfortably in a chair and leaning against a backrest. The validity of this model was verified by comparing the estimated values and the actual values measured using a force plate.

The results of the present study show that the value of the shear force measured using a force plate 
$(35.9 \pm 15.8[\mathrm{~N}])$ was similar to the value of the shear force estimated using the experimental model $(36.6 \pm 11.8[\mathrm{~N}])$, and exhibited a strong positive correlation. These results show the validity of the experimental model for the upper body. However, the model did not take into account the legs, and this issue was addressed by subtracting the leg friction force. In preliminary experiments prior to this study, we measured the ground reaction force (GRF) to the soles of the feet using a force plate. The vertical GRF was $18.6 \pm 2.2 \%$ body weight (BW), and the posterior horizontal GRF was $2.2 \pm$ $0.6 \% \mathrm{BW}$. These results showed that GRFs at the soles of the feet depend on knee flexion angles, and flexing the knee at 90 degrees while sitting comfortably could decrease the horizontal GRF to a negligible value. Therefore, by adjusting the flexion angle of the knee, the reaction force to the foot does not markedly affect the estimation of shear force. Therefore, while sitting comfortably in a chair, the rotational moment in the posterior direction created by leaning of the upper body is opposed by leaning against the backrest, and the subsequent shear force in the anterior direction can be estimated. Because the shear force in a comfortable sitting posture could easily be estimated without using a special device, this capability will be useful in clinical settings.

While sitting comfortably in a chair, the posterior inclination of the trunk and pelvis increases. In the sacral sitting posture, the center-of-gravity line deviates from the sitting pressure center (action point), and a large posterior-direction rotational moment is generated in the trunk region ${ }^{11)}$. Subsequently, shear force is generated in the gluteal region. Furthermore, pressure that is divided into two by the left and right ischial bones concentrates on one side, thus resulting in very strong pressure. Therefore, a comfortable sacral sitting posture has a higher risk for a pressure ulcer from the viewpoints of the pressure and mechanical factors of the shear force. Empirical evidence in the higher onset risk for pressure ulcers in the sacral and caudal regions in clinical settings ${ }^{10)}$ supports this view. Thus, shear force estimated by the experimental model may aid the prevention of pressure ulcers.

The present experimental model makes it possible to estimate the shear force for a person sitting in a comfortable posture using a relatively simple formula. In the future, we plan to investigate the effects of wrinkling of the wheelchair-seating surface and cushions on shear force by measuring static friction coefficients and the maximum friction force on the body in the sitting posture.

\section{ACKNOWLEDGEMENTS}

This study was supported by a grant-in-aid from the 2006 Kawasaki University of Medical Welfare's expense budget for medical welfare study and research.

\section{REFERENCES}

1) Dairiki H, Fujii M, Awa K, et al.: Study of generation mechanism and pressure sore by shear force. JSME, 2004, 4: 217-218.

2) Trefler E, Hobson DA, Taylor SJ, et al.: Seating and mobility for person with physical disabilities. 1st ed. Memphis: Therapy skill builders, 1993, pp3-5.

3) Kobara K, Eguchi A, Watanabe S: Relationships between backrest of a chair and the position of pelvis on the peak load value at the ischium and the shear stress -An estimate of the shear stress with an experiment model-. Rigakuryouho Kagaku, 2006, 21: 293-297.

4) Gilsdorf $P$, Patterson R, Fisher $S$, et al.: Sitting forces and wheelchair mechanics. J Rehabil Res Dev, 1990, 27: 239-246.

5) Goossens RHM, Snijders CJ, Holscher TG, et al.: Shear stress measured on beds and wheelchairs. Scand J Rehab Med, 1997, 29: 131-136.

6) Nakamura R, Saito H, Nagasaki H: Fundamental kinesiology 3rd ed. Tokyo: Ishiyaku Publishers, 2005, pp332-336.

7) Kubo Y, Yamaguchi M, Ohno N, et al.: Investigation of the validity of visual assessment of center of gravity in the analysis of posture and movement. Rigaku ryohogaku, 2006, 33: 112-117.

8) Sakuta Y, Takahashi M: The interaction between pressure and shear force as a factor of pressure ulcers by assessing blood flow measurement. JSMBE, 2006, 44: 101-106.

9) Nojima Y, Nakano K, Takahashi M, et al.: Stress analysis of subcutaneous tissue in use of various cushion materials. Jpn J PU, 2005, 7: 785-791.

10) Kikuchi M, Kai Y, Nakai N, et al.: Control of depressurization and seating are effective for a case of ischial pressure ulcer. Skin Research, 2005, 4: 448452.

11) Ehara Y, Yamamoto S: Introduction to bodydynamics. Analysis of gait and gait initiation. 1st ed. Tokyo: Ishiyaku Publishers, 2004, pp2-4. 